\title{
Formal Analysis and Application of the New Mode of "VR+ Education"
}

\author{
Xiangyu Meng \\ Dalian Naval Academy of the PLA, Liaoning, Dalian 116018
}

\begin{abstract}
VR+ education" is the development trend of educational technology in the future. Aiming at the difficult problem of combining virtual reality technology with educational technology, and based on the purpose of adapting to the future development needs, this paper uses formal logic method to analyze the promotion conditions of "VR+education", and puts forward the application strategies and impact evaluation, which can provide reference for the practical application of the new model in the field of teaching.
\end{abstract}

\section{Conceptual simplicity and extension}

VR (Virtual Reality) is a computer simulation system for creating and experiencing virtual world. It can simulate environment, multi-source information fusion, interactive three-dimensional dynamic scene and entity behavior simulation. The "education" here refers specifically to educational technology, which refers to the application of modern science and technology in education. Formal method is to compare and find out the ways of interlinking different forms of thinking in logic science. It can reveal all kinds of logic rules accurately, formulate corresponding logic rules, make all kinds of theoretical systems more rigorous, and train thinking correctly and improve the abstract ability of thinking. The application of VR technology in higher education and teaching is the inevitable outcome of the development of modern information-based teaching. The first dimension is that the initial way of learning only depends on reading and memorizing knowledge points. Then multimedia teaching enters the classroom and brings slides and videos into learning, which is the second dimension. Now VR can be regarded as the third dimension. Dimensions, namely experiential learning, are richer and more immersive than previous video teaching. VR can make the learning effect of learners further on the basis of multimedia teaching, which is obvious in theory. Based on this, the new model of "VR + education" comes along with the development of science and technology and education concept. What strategies should be used and how to evaluate the effect should be the important direction of current education research to promote the development of "VR + education".

\section{Analysis of the conditions for comprehensively promoting "VR+ education"}

\footnotetext{
a Corresponding author: likandl@126.com
}

Through the analysis and Research on the development of modern educational technology and virtual reality technology by predecessors, it is not difficult to find that virtual reality technology has distinct and unique advantages in the field of education, which provides effective help for the innovation of educational technology means and meets most of the new teaching requirements of modern schools. It can provide learners with free learning space and stimulate learners' interest and enthusiasm in learning. VR (virtual reality) refers to the technology of providing immersion feeling in the interactive three-dimensional environment generated on the computer by utilizing computer graphics system and various interface devices such as reality and control. It uses computer to generate a simulation environment. It is an interactive three-dimensional dynamic scene and entity behaviour system simulation based on multi-source information fusion, which immerses users in the environment. If VR technology is interpreted popularly, it is to use computer technology to help you realize your "daydream". In this process, VR technology will simulate a realistic three-dimensional virtual world, and through vision, hearing, smell, touch, taste and so on, let learners feel the effect of immersion. Virtual reality technology has the advantages that traditional teaching methods do not possess. Firstly, it is reflected in the creation of situations. Virtual reality technology can create a realistic and intuitive learning environment for learners, facilitate learners' understanding and grasp of knowledge, fully mobilize learners' enthusiasm and initiative in learning, and strengthen learners' understanding of knowledge. It is important to grasp knowledge and cultivate learner's ability of autonomous learning. At the same time, the creation of virtual scene is conducive to enhancing learners' innovation and thinking. Through the application of virtual reality technology, learners can choose their own learning mode, conversational situation, teaching content, etc. The computer also provides relevant 
feedback for learners to enhance their interest in thinking and research. In addition, virtual reality technology can make up for the shortcomings of traditional teaching methods, minimize the obstacles caused by security, economy and other issues. Through virtual reality technology, rare natural landscape can be displayed, some experimental dangers can be avoided, and can be reused. For example, in chemical experiments, operators can simulate explosion, combustion and other high-risk phenomena through virtual technology; in physical experiments, they can experience the sense of weightlessness, or witness nuclear fusion, fission and other scenarios; in geographical learning, they can experience the changes of four seasons, crustal movement and other feelings; and use virtual reality. Real technology can also enable learners to easily learn automobile or even aircraft driving technology, in order to avoid serious accidents caused by operational errors, or to conduct simulated surgery, saving medical experimental resources and avoiding unnecessary losses. The new technology of virtual reality VR, which combines puzzle games, situational learning, collaborative learning, distance education and other characteristics, will solve many previously unsolvable educational problems, and let us feel all-round education. In short, the application of virtual reality technology in modern education can improve the level of education, enrich teaching means, provide better learning environment for learners, avoid accidents and waste of resources, and have many practical advantages. It is urgent to promote the demand of "VR+education" in an all-round way.

\section{The strategy and impact assessment of "VR+ education"}

Education meets VR, which brings high immersion learning experience and efficiency to learners. In order to make a perfect combination of new technology and education, we need to make clear the use of strategy and impact assessment.

\subsection{Promoting learner autonomy and learning}

The application of virtual reality technology in modern education can promote learners to study and explore independently, and use human-computer interaction technology to lead learners to appreciate different artistic conceptions or watch different phenomena. Learners can use virtual teaching system to experience the rare natural phenomena, beautiful scenery, and even the change and development process of things in reality. They can also use virtual reality technology to make some profound and obscure knowledge intuitive and visual, in order to deepen the understanding of theory. At the same time, the use of virtual reality technology can break the barriers in time and space. The experiments that need to be observed for a long time in the past can be solved in one class by simulating the scene, which greatly saves the time and energy of learners, but the teaching effect is not inferior. It is of great significance for learners to find the pleasure of learning in the virtual scene, and to conduct research and discussion more actively, so as to create a good atmosphere for learning and improve the overall teaching level.

\subsection{In order to improve the effect of simulation experiment scene}

Using virtual reality technology to simulate experiments is the most widely used aspect of its advantages in modern education. Educators can use virtual reality technology to set up laboratories such as physics, chemistry, biology, medicine and so on, which not only facilitates teachers to explain different contents, but also deepens learners 'understanding of obscure subject knowledge. At present, virtual laboratory is widely used in science teaching. Learners can carry out geographic experiments such as earthquake, volcanic eruption, or biological experiments such as human anatomy in the laboratory. Virtual reality technology also has great potential in the field of literature and history education, such as the establishment of virtual historical figures in the history virtual laboratory, allowing learners to cross time and space, communicate directly with historical figures, stimulating novelty while deepening learners 'perception of historical phenomena.

\subsection{To enhance learners' operational skills as a means}

Using the characteristics of multi-perception, interaction and presence of virtual reality technology, the learners are encouraged to play roles in virtual situations, thus devoting themselves wholeheartedly to practice and skills enhancement, which is very beneficial to the enhancement of learners 'various skills. In the simulation scenario, learners can conduct various practical skills training at low cost, such as architectural design skills, military co-ordination skills, medical skills, driving skills, machine repair skills, planting skills and so on. Virtual training is widely concerned and praised in modern education because of its low cost and no danger. Teachers can save a lot of effort to discuss the teaching plan, learners also have an additional platform and environment for practice, and can continue to train in private space until they master the relevant operational skills.

\subsection{Make up for distance education and special education}

With the development of satellite communication technology and the continuous popularity of the Internet, the development of distance education in China has gradually entered the orbit, and some difficulties have arisen concerning the specific teaching mode. Virtual reality technology can just make up for the shortcomings in this regard and provide a good learning environment for distance education. On the one hand, through the network virtual classroom, virtual laboratory, library and other mechanisms, learners can choose the courses of interest independently. On the other hand, virtual reality 
technology can also play a great role in the special education of the disabled. Deaf-mute people can convert gestures into sounds through glove sensors to facilitate normal communication; blind people can use eye tracker to translate eyes into language; and mentally retarded children can explore and familiarize themselves with the world through three-dimensional image and training system constructed by virtual reality technology.

\section{Concluding remarks}

With the continuous development and improvement of science and technology, many new technologies have sprung up. As a leading technology, the application and development of VR technology has attracted worldwide attention and attention. In order to meet the needs of future development, the education industry has begun to attempt the new model of "VR + education", to carry out formal analysis, application strategies and impact evaluation of the new model, and to lay a theoretical foundation for its good results in teaching, training and military education. It is very necessary to analyze the application of VR technology in the field of education. The exploration, application and theoretical research of the new mode of "VR+ education" is an important development direction in the field of education.

\section{References}

1. Huang Yiyu. Overview of the Application of Virtual Reality (VR) in Education. China's Education Informatization, 2018 (1): 11-16.

2. Liu Dejian, Liu Xiaolin, Zhang Yan. Potential Progress and Challenges in the Application of Virtual Reality Technology in Education. Open Education Research, 2016 (4): 25-31.

3. Cai Su, Zhang Hao. VR/AR Education Application Case and Development Trend. Digital Education, 2017 (3): 1-10. 